Liver, Pancreas and Biliary Tract

\title{
Hepatitis B virus related cryoglobulinemic vasculitis: A multicentre open label study from the Gruppo Italiano di Studio delle Crioglobulinemie - GISC
}

\author{
Cesare Mazzaro ${ }^{\mathrm{a}, *}$, Luigino Dal Maso ${ }^{\mathrm{b}, * *}$, Teresa Urraro ${ }^{\mathrm{c}}$, Endri Mauro ${ }^{\mathrm{d}}$, \\ Laura Castelnovo $^{\mathrm{e}}$, Pietro Casarin ${ }^{\mathrm{d}}$, Giuseppe Monti ${ }^{\mathrm{e}}$, Valter Gattei ${ }^{\mathrm{a}}$, \\ Anna Linda Zignego ${ }^{c}$, Gabriele Pozzato ${ }^{\mathrm{f}}$ \\ ${ }^{a}$ Clinical and Experimental Onco-Hematology Unit, CRO Aviano National Cancer Institute, Aviano, Italy \\ ${ }^{\mathrm{b}}$ Epidemiology and Biostatistics Unit, CRO-Aviano National Cancer Institute, Aviano, Italy

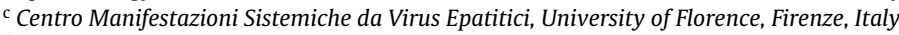 \\ d Department of Internal Medicine, Pordenone General Hospital, Pordenone, Italy \\ e Department of Internal Medicine, Saronno General Hospital, Saronno (VA), Italy \\ ${ }^{\mathrm{f}}$ Department of Medical and Surgical Sciences, University of Trieste, Trieste, Italy
}

\section{A R T I C L E I N F O}

\section{Article history:}

Received 10 November 2015

Accepted 29 March 2016

Available online 2 April 2016

\section{Keywords:}

Cryoglobulinemia

Entecavir

Hepatitis B virus

Vasculitis

\begin{abstract}
A B S T R A C T
Background: Cryoglobulinemic vasculitis (CV) related to Hepatitis-B Virus (HBV) is rare and its treatment is ill-defined.

Aims: To describe clinical and treatment characteristics of HBV-related CV patients. In addition, the efficacy of treatment with antiviral agent nucleotide (NUC), including Entecavir, Adefovir, and Lamivudine, was explored.

Methods: In four Italian centres, 17 HBV-positive CV patients (median age 56 years, range 45-70) were enrolled.

Results: The extrahepatic manifestations were: purpura (100\%), arthralgias (71\%), peripheral neuropathy (29\%), chronic hepatitis (47\%), liver cirrhosis (29\%), and glomerulonephritis (18\%). Mixed cryoglobulinemias were type II ( $88 \%$ ) and type III (12\%). The median cryocrit was 3\% (range 1-14), rheumatoid factor was $200 \mathrm{U} / \mathrm{L}$ (range 20-5850), C4 was $12 \mathrm{mg} / \mathrm{dl}$ (range 2-31), ALT $71 \mathrm{U} / \mathrm{L}$ (range 36-114). All patients were HBsAg-positive and $80 \%$ anti-HbeAg-positive. At enrollment, they were treated with steroids (eight), Entecavir (five), Alpha-IFN (two), Adefovir and Lamivudine (one each). After NUC treatment, no disease progression was observed and, in all patients, HBV-DNA became undetectable. Moreover, a regression of purpura and a reduction of cryocrit were observed. Four patients died during therapy, two of kidney failure and two of liver cirrhosis.

Conclusion: NUC therapy appeared to be safe and effective in CV-related HBV.
\end{abstract}

(c) 2016 Editrice Gastroenterologica Italiana S.r.l. Published by Elsevier Ltd. All rights reserved.

\section{Introduction}

Infection with hepatitis $\mathrm{B}(\mathrm{HBV})$ is a serious worldwide public health problem. It is estimated that about 350 million people are

\footnotetext{
* Corresponding author at: Clinical and Experimental Onco-Hematology Unit, CRO Aviano National Cancer Institute IRCCS, Via Franco Gallini 2, Aviano, Pordenone 33081, Italy.

** Corresponding author at: Epidemiology and Biostatistics Unit, CRO Aviano National Cancer Institute IRCCS, Via Franco Gallini 2, Aviano, Pordenone 33081, Italy.

E-mail addresses: cesare.mazzaro@gmail.com (C. Mazzaro),

epidemiology@cro.it (L. Dal Maso).
}

chronically infected with HBV. The clinical manifestations of HBV range from acute or fulminant hepatitis to various forms of chronic infection, including an inactive carrier state, chronic hepatitis, cirrhosis, and hepatocellular carcinoma [1,2].

About $20 \%$ of HBV patients develop extrahepatic manifestations; among them, polyarthritis nodosa and glomerulonephritis are the most severe. Other manifestations (e.g., dermatitis, polyarthralgias and arthritis, respiratory disease, aplastic anemia) are rare and cryoglobulinemic vasculitis (CV) occurs in approximately $3 \%$ of patients $[3,4]$. No association has been found between these manifestations and viral genotypes [3,4]. Reported cases of HBVrelated CV are rare, the most effective treatment is still unknown, as no definitive guidelines have been issued to date. The conventional 
immunosuppressive therapy used in rheumatologic disorders is not indicated in HBV-related CV, while antiviral therapy with nucleotide analogues seems to be the best treatment in these cases. Evidence on the efficacy of the antiviral treatment of HBVrelated CV with nucleoside analogues is limited, since only case reports are presently available in the literature [5-11]. According to Brouet et al. [12], the cryoglobulinemias are classified in three types. In type I, the cryoglobulins are formed by monoclonal immunoglobulins only, commonly IgM. This type of cryoglobulins are associated with lymphoproliferative disorder (multiple myeloma or Waldenström's disease). In types II and III (so called mixed cryoglobulinemias, MC), the cryoglobulins are immunecomplexes composed by polyclonal IgGs, the antigen(s), and monoclonal or policlonal IgMs, respectively. The IgM are endowed with rheumatoid factor activity, i.e., against polyclonal IgG [12-14]. Types II and III are associated with chronic viral infections (i.e., $\mathrm{HBV}$ and $\mathrm{HCV}$ ), connective tissue diseases, and lymphoproliferative disorders.

The clinical manifestations of MC are due to the deposition of immune-complexes in several organs and tissues. MC may determine not only purpura, arthralgias and asthenia, but also more serious lesions of the skin (large ulcers), and neurologic and renal involvement [15-19]. The therapy of MC is based on immunosuppressive agents (steroids, cyclophosphamide, azathioprine, cyclosporine) for patients with mild-to-moderate disease, whereas more aggressive treatments, such as corticosteroids or plasmapheresis or high-dose cyclophosphamide, are needed in patients with severe disease [20,21]. More recently, data on the efficacy and safety of anti-CD20 monoclonal antibodies in MC vasculitis have emerged [22,23].

The purpose of this retrospective study was to assess the treatment in a group of patients with HBV-related CV.

\section{Patients and methods}

Seventeen consecutive patients (10 females and 7 males) affected by HBV-related CV were included in this study. All cases were enrolled between 2006 and December 2014 at the Department of Internal Medicine of Pordenone General Hospital, at the 'Centro Manifestazioni Sistemiche da Virus Epatitici', University of Florence, and at the Department of Internal Medicine of the Saronno General Hospital.

The inclusion criteria of this retrospective study was the presence of chronic HBV associated with clinical symptoms of CV. All patients were positive for HBV surface antigen (HBsAg) and measurable levels of serum HBV-DNA were detectable. The patients showing anti-Hepatitis $C$ and/or anti-human immunodeficiency virus (HIV) antibodies or with concomitant malignant diseases were excluded.

Clinical and biological data were recorded for each patient at onset, during follow-up, and at the end of follow-up. The visits were scheduled every 3 months and information collected until June 2015. Liver function as well as kidney function testing and haematological parameters, laboratory assessment, including determination of complement components, rheumatoid factor and cryoglobulin serum levels were carried out using standard methods. MC was classified as type II in presence of monoclonal IgM complexed with polyclonal IgG, and type III in presence of polyclonal immunoglobulins. Patients showing elevated creatinine levels and $24 \mathrm{~h}$ proteinuria underwent biopsy for kidney involvement. Liver biopsies were performed in patients showing only signs of chronic liver disease. The grading for liver fibrosis was performed by means of transient elastography [24].
Table 1

Baseline, clinical, biochemical and histological features of 17 patients with HBV positive cryoglobulinemic vasculitis. ${ }^{\text {a }}$

\begin{tabular}{lcc}
\hline & Median (range) & $N(\%)$ \\
\hline Males & & $7(41 \%)$ \\
Type II cryoglobulinemia & $56(45-70)$ & $15(88 \%)$ \\
Age & $71(36-114)$ & \\
ALT $(6-78 \mathrm{U} / \mathrm{L})^{\mathrm{b}}$ & $1.0(0.7-2.4)$ & \\
Creatinine $(0.50-1 \mathrm{mg} / \mathrm{dl})^{\mathrm{b}}$ & $3.0(1.0-14.0)$ & \\
Cryocrit $(\%)$ & $200(20-5850)$ & \\
Rheumatoid factor $(0-25 \mathrm{IU} / \mathrm{ml})^{\mathrm{b}}$ & $12(2-31)$ & \\
C4 $(10-40 \mathrm{mg} / \mathrm{dl})^{\mathrm{b}}$ & & $17(100 \%)$ \\
Clinical manifestations & & $12(71 \%)$ \\
Purpura & & $1(6 \%)$ \\
Arthralgias & & $5(29 \%)$ \\
Leg ulcer & & $3(18 \%)$ \\
Peripheral neuropathy & & $3(18 \%)$ \\
Raynaud phenomenon & & $8(47 \%)$ \\
Glomerulonephritis & & \\
Chronic hepatitis & & \\
Liver cirrhosis & & \\
\hline
\end{tabular}

ALT, alanine aminotransferase.

a Followed for a median time of observation of 5.2 years (range 0.5-19 years).

b Normal values.

\subsection{Treatment}

Due to the retrospective nature of our study, the included patients had received different treatments. High-dose corticosteroids $(1-10 \mathrm{~m} / \mathrm{kg}$ ) were administered to treat systemic vasculitis and critical manifestations of MC (renal, neurological and hyperviscosity syndromes) [21]. Low-medium corticosteroid doses $(0.1-0.5 \mathrm{mg} / \mathrm{kg} /$ day) were used in mild or moderate cryoglobulinemic vasculitis (purpura, arthralgias, peripheral neuropathy) [21]. Antiviral therapy was also not homogeneous; indeed, some patients were treated with interferon-alpha-2b (5MU/die for 6 months), others with Entecavir $0.5 \mathrm{mg} /$ day, or with Adefovir $10 \mathrm{mg} /$ die and even with Lamivudine $100 \mathrm{mg} /$ die. Biochemical and clinical parameters were measured every two months during treatment, while HBsAg, HBV-DNA, cryoglobulins, RF and complement fraction C4 every 6 months.

\subsection{Outcome measures}

As previously reported [25], four types of response to treatment were initially defined (virological, biochemical, immunological, and clinical) and herein presented at 48 months of continuous treatment (after enrollment).

\subsection{Statistical analysis}

Descriptive statistics of relevant variables were performed using median and range. Mean and standard deviation (SD) were not informative and not presented because of the limited number of patients.

\section{Results}

\subsection{Patients' characteristics}

The study enrolled 17 patients (seven males, 41\%), median age 56 (range 45-70 years) with HBV-related CV. Table 1 shows patients' biochemical, clinical, and histological characteristics. All of them presented purpura on the leg and asthenia, and 12 of them (71\%) also arthralgias. Peripheral neuropathy was found in five cases (29\%), and one of them showed a large ulcer on the left leg (6\%). Fifteen cases (88\%) had type II MC and two cases 
Table 2

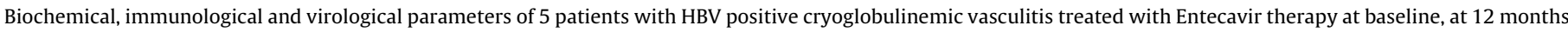
and at 48 months of therapy.

\begin{tabular}{|c|c|c|c|}
\hline & Baseline & At 12 months & At 48 months \\
\hline \multirow[t]{2}{*}{ Purpura positive } & 5 & 0 & 0 \\
\hline & Median (range) & Median (range) & Median (range) \\
\hline Mean ALT $(6-78 \mathrm{U} / \mathrm{L})^{\mathrm{a}}$ & $71(39-82)$ & $17(13-40)$ & $26(13-33)$ \\
\hline Creatinine $(0.50-1 \mathrm{mg} / \mathrm{dl})^{\mathrm{a}}$ & $1.0(0.7-1.2)$ & $0.7(0.5-1.0)$ & $0.8(0.6-1.2)$ \\
\hline Cryocrit (\%) & $4(2-9)$ & $1(0-3)$ & $1(0-3)$ \\
\hline Rheumatoid factor $(0-25 \mathrm{IU} / \mathrm{ml})^{\mathrm{a}}$ & $119(88-5850)$ & $80(31-5272)$ & $82(20-5026)$ \\
\hline $\mathrm{C} 4(10-40 \mathrm{mg} / \mathrm{dl})^{\mathrm{a}}$ & $8(4-31)$ & $7(1-10)$ & $10(2-24)$ \\
\hline HBV-DNA (IU/ml) & $2265(1540-4020)$ & $<20(-)$ & $<20(-)$ \\
\hline Undetectable HBV & 0 & $5(100 \%)$ & $5(100 \%)$ \\
\hline
\end{tabular}

ALT, alanine aminotransferase. HBV, hepatitis B virus.

a Normal values.

(12\%) type III MC. Cryocrit ranged from 1\% to $14 \%$ (median 3\%), serum complement $\mathrm{C} 4$ levels were low in all cases (median 12; range: $2-31 \mathrm{mg} / \mathrm{dl}$ ), and serum levels of rheumatoid factor (RF) were higher than recommended in 14 cases (82\%), median 200 (range: $20-5850 \mathrm{IU} / \mathrm{L}$ ). ALT was elevated in 14 cases $(82 \%)$ with median $=71$ (range: $36-114 \mathrm{U} / \mathrm{L}$ ), serum creatinine levels were abnormal ( $>1 \mathrm{mg} / \mathrm{dl}$ ) in three cases (18\%), proteinuria in the $24 \mathrm{~h}$ was elevated in three cases (18\%), ranging from 3 to $5 \mathrm{~g} / 24 \mathrm{~h}$. Renal biopsy showed the presence of membrane proliferative glomerulonephritis type I in all three cases (18\%). All patients showed positive test results for $\mathrm{HBsAg}$ and antiHbeAg, whereas test results for anti-HBsAg, HBeAg were negative. HBV-DNA was tested in five cases treated with Entecavir. The median value was 2265 $(1540-4020 \mathrm{IU} / \mathrm{ml})$.

\subsection{Effect of therapeutic regimen on response to treatment and outcome}

All patients were suitable for therapeutic regimens; in particular, therapeutic schedules were as follows: corticosteroid, in eight cases; Entecavir, in five cases; Interferon-alpha, in two cases; and Adefovir and Lamivudine, in one case each. Two patients with $\mathrm{HBV}$-related $\mathrm{CV}$ and glomerulonephritis treated with Interferon-alpha $5 \mathrm{MU} /$ day for 6 months, did not obtain clinical response of the purpura and arthralgias. Since their levels of cryocrit, RF, complement fraction C4, ALT, creatinine, proteinuria/24h remained unchanged, they were considered as non-responders. After subsequent treatment with low-dose corticosteroid, both patients obtained clinical and immunological response but, as expected, no virological response. Five additional patients were treated with low-dose corticosteroid for 48 months: purpura and arthralgias regressed in three patients; while clinical symptoms persisted in two cases after the end of treatment.

Corticosteroid therapy induced an improvement of vasculitis (i.e., purpura: eight positive at enrolment; two at 12 months; and four at 48 months) and a reduction of cryocrit levels in all patients (median $=3 \%$ at enrolment, $1.5 \%$ after 48 months), but no patient showed undetectable levels of cryoglobulins at the end of treatment (data not shown). The RF level remained elevated after treatment in all patients. C4 serum levels remained unchanged (median $=12 \mathrm{mg} / \mathrm{dl}$ ) at the end of treatment in all patients. No virological response was observed in five patients treated with corticosteroids who had not received antiviral therapy. At the end of treatment with corticosteroids, a high median level of ALT was observed $(62 \mathrm{U} / \mathrm{L})$, unchanged in comparison with that at enrolment ( median $=66$ ).

\subsection{Virological response with NUC (Entecavir, Adefovir and Lamivudine)}

During treatment with Entecavir in five patients (Table 2), Adefovir in one patient and Lamivudine in another, HBV-DNA had undetectable viremia in all of them (100\%), after 12 months of therapy. At the end of therapy, all patients maintained undetectable viremia, while HBsAg remained positive in all of them.

\subsection{Clinical response with NUC}

Purpura regressed at the end of therapy in all patients treated with Entecavir (Table 2). An improvement of arthralgias was observed in three patients at the end of treatment. The ulcer on the leg regressed in one patient, at the end therapy. Purpura and arthralgias regressed in one patient treated with Adefovir and in another patient treated with Lamivudine.

\subsection{Immunological response with NUC}

Entecavir induced a reduction of cryocrit levels in all cases, but only two cases showed undetectable levels of cryoglobulins at the end of treatment (Table 2). The RF levels decreased in all patients, but a normal RF was observed in only one case at the end of therapy and at follow-up (Table 2). C4 serum levels remained low during treatment and at the end of therapy. A normal C4 serum level was not observed in all patients, at the end of therapy.

In one patient, treated with Adefovir, cryocrit disappeared from serum, from $5 \%$ to $0 \%$, RF levels decreased from 200 to 140 and $120 \mathrm{U} / \mathrm{L}$ during treatment and at the end of therapy. C4 serum level remained low from 8 to 6 and $8 \mathrm{mg} / \mathrm{dl}$ during treatment and at the end of therapy.

In the patient treated with Lamivudine, cryocrit regressed in serum, from $14 \%$ to $12 \%$ and $12 \%$, RF regressed from 2900 to 2490 and $2900 \mathrm{U} / \mathrm{L}, \mathrm{C} 4$ serum level remained low from 12 to 11 and $11 \mathrm{mg} / \mathrm{dl}$ during treatment and the end of therapy.

\subsection{Biochemical response with NUC}

Entecavir induced an ALT normalization in four patients after 12 months of treatment (Table 2). No exacerbations were observed. Adefovir induced an ALT normalization from 85 to 30 and 34 and $\mathrm{U} / \mathrm{L}$ at the end of therapy and at follow-up. Lamivudine induced an ALT normalization from 78 to 20 and 21 and U/L after 12 months of treatment.

At the last follow-up visit, in December 2014, all patients continued the treatment with Entecavir, Adefovir and Lamivudine. During therapy, adverse events were not observed. In this group, 
five patients died (29\%); the causes of death were cirrhosis complications, ascites, and encephalopathy (two cases), hepatocellular carcinoma in cirrhosis, sepsis in kidney failure (one case), and kidney and heart failure (one case).

\section{Discussion}

HBV-related CV is rare as compared to HCV-related CV, and the treatment is still poorly understood. Antiviral therapy, mostly based on mono-therapy with NUC such as Lamivudine [5,6,9], Adefovir Dipivoxil [26], Entecavir [8], Telbivudine [10], or Tenofovir [40] has given encouraging results in terms of viral clearance and clinical remission in HBV-related CV. Many studies have suggested a possible role of $\mathrm{HBV}$ replication in the $\mathrm{CV}$ pathogenesis and a relationship between undetectable HBV-DNA and regression of CV after antiviral therapy [5-11]. Our study is in agreement with previous results, showing multiple organ involvement (skin, liver, and kidney), and with the beneficial effect of therapy with Entecavir, Adefovir, and Lamivudine on clinical, immunological and virological parameters. Thus, a role of HBV infection in cryoglobulinemia emerged.

The severity of hepatic and extrahepatic manifestations prompted us to start antiviral therapy, leading to a regression of purpura, a decrease of cryocrit, and HBV-DNA became undetectable in all patients. In this study, Interferon-alfa was ineffective for clinical symptoms of vasculitis and suppression of HBV. While corticosteroid therapy was effective for clinical symptoms of vasculitis, it was ineffective for suppression of HBV viremia and immunological features. The patients with HBV related nephropathy were non-responders to Interferon-alfa and corticosteroids therapy. In this study, no patient treated with NUC was affected by HBV related nephropathy.

Renal involvement in MC showed a poor prognosis, as previously reported $[27,28]$. NUC have also been administered with some benefit to treat HBV-related nephropathy $[29,30]$. Recently, new findings on the efficacy and safety of anti-CD20 monoclonal antibodies and Interferon in MC with glomerulonephritis have emerged [31,32]. In this study, two patients died of kidney failure, proving this poor prognosis. The observation that therapy with NUC in HBVrelated $\mathrm{CV}$ was without side effects indicated that compliance is promising for these patients.

Concerning the treatment of $\mathrm{HBV}$ related $\mathrm{CV}$, several questions remain unanswered about the proper time to initiate antiviral therapy and the suitable duration. The goal of HBV therapy should be viral suppression, although this may require a long-term therapy in chronic hepatitis. In patients with $\mathrm{HBV}$ related $\mathrm{CV}$, antiviral therapy may be discontinued after persistent $\mathrm{HBs} A g$ seroconversion in antiHBs and undetectable HBV-DNA, as recommended by international guidelines [33]. Rates of HBs-Ag loss following 12 months of treatment were 0\% with Lamivudine, Adefovir, and Entecavir [34,35]. Hbs-Ag loss rate increased to $9 \%$ at three years and $12 \%$ at five years following Peginterferon alpha-2a therapy [36,37]. In contrast, HBs$\mathrm{Ag}$ loss was rarely observed during the first four-to-five years of NUC therapy in HBe-negative patients [38,39].

In conclusion, our study demonstrated the effectiveness and safety of therapy with NUC for HBV-related CV. Since the sample size of this study was small, large-scale cooperative studies are needed to assess this issue.

\section{Conflict of interest}

None declared.

\section{Acknowledgment}

The authors wish to thank Mrs. Luigina Mei for editorial assistance.

\section{References}

[1] Gamen D, Prince AM. Hepatitis B virus infection - natural history and clinical consequences. New England Journal of Medicine 2004;350:1118-29.

[2] Wright TL, Lau JY. Clinical aspects of hepatitis B virus infection. Lancet 1993;342:1340-4

[3] Cacoub P, Sadoun D, Bourliere M, et al. Hepatitis B virus genotypes and extrahepatic manifestations. Journal of Hepatology 2005;43:764-70.

[4] Cacoub P, Terrier B. Hepatitis B-related autoimmune manifestations. Rheumatic Disease Clinics of North America 2009;35:125-37.

[5] Filer A, Hughes A, Kane K, et al. Successful treatment of hepatitis B-associated vasculitis using lamivudine as the sole therapeutic agent. Rheumatology 2001;40:1064-5

[6] Stecevic J, Pevzner M, Gordon S. Successful treatment of hepatitis B-associated vasculitis with lamivudine. Journal of Clinical Gastroenterology 2003;36:451.

[7] Sawabe T, Uenotsuchi T, Imafuku S, et al. Remission of hepatitis B virus-related vasculitis with lamivudine. Annals of Internal Medicine 2004;140:672-3.

[8] Enomoto M, Makanishi T, Ishii M, et al. Entecavir to treat hepatitis B-associate cryoglobulinemic vasculitis. Annals of Internal Medicine 2008;149:912-3.

[9] Conca P, Riccio A, Tarantino G. Successful lamivudine monotherapy in an elderly patient suffering from HBV-related decompensated cirrhosis associated with widespread leukocytoclastic vasculitis. International Journal of Immunopathology and Pharmacology 2009;22:531-5.

[10] Boglione L, D'Avolio A, Cariti G, et al. Tellbivudine in treatment of hepatitis B-associated cryoglobulinemia. Journal of Clinical Virology 2013;56:167-9.

[11] Vigano M, Martin P, Cappelletti M, et al. HBV-associated cryoglobulinemic vasculitis: remission after antiviral therapy with Entecavir. Kidney and Blood Pressure Research 2014;39:65-73.

[12] Brouet JC, Cauvel JP, Danon F, et al. Biologic and clinical significance of cryo globulins. A report of 86 cases. American Journal of Medicine 1974:57:775-88.

[13] Gorevicc PD, Frangione B. Mixed cryoglobulinemia cross-reactive idiotypes: implication for the relationship of MC to rheumatic and lymphoproliferative diseases. Seminars in Hematology 1991;28:79-94.

[14] Ferri C, Zignego AL, Giuggioli D, et al. HCV and cryoglobulinemic vasculitis. Cleveland Clinic Journal of Medicine 2002;69(Suppl. 2):SII20-3.

[15] Cacoub P, Gragnani L, Comarmond C, et al. Extrahepatic manifestations associated with hepatitis C virus infection. Digestive and Liver Disease 2014;46:S165-73.

[16] Johnson RJ, Grecht DR, Yamabe H, et al. Membranoproliferative glomerulonephritis associated with hepatitis C virus infection. New England Journal of Medicine 1993:328:465-70.

[17] Yamabe H, Johnson RJ, Grecht DR, et al. Hepatitis C virus infection and membrano-proliferative glomerulonephritis in Japan. Journal of the American Society of Nephrology 1995;6:220-3.

[18] Fabrizi F, Lunghi G, Messa P, et al. Therapy of hepatitis C virus associated glomerulonephritis: current approaches. Journal of Nephrology 2008;21:813-25.

[19] Perico N, Cattaneo D, Bikbov B, et al. Hepatitis C infection and chronic renal disease. Clinical Journal of the American Society of Nephrology 2009;4:207-8.

[20] Mazzaro C, Monti G, Saccardo F, et al. Efficacy and safety of peginterferon alpha-2b plus ribavirin for HCV-positive mixed cryoglobulinemia: a multicentre open-label study. Clinical and Experimental Rheumatology 2011;29: 933-41.

[21] Pietrogrande M, De Vita S, Zignego AL, et al. Recommendations for the management of mixed cryoglobulinemia syndrome in hepatitis C virus-infected 215 patients. Autoimmunity Reviews 2011;10:444-54.

[22] Zaja F, De Vita S, Mazzaro C, et al. Efficacy and safety of rituximabin type Il mixed cryoglobulinemia. Blood 2003:15:101-10.

[23] Sansonno D, De Re V, Lauletta G, et al. Monoclonal antibody treatment of mixed cryoglobulinemia resistant to interferon alpha with an anti-CD20. Blood 2003; 101:3818-26

[24] Hagan M, Asrani SK, Talwalkar J. Non-invasive assessment of liver fibrosis and prognosis. Expert Review of Gastroenterology \& Hepatology 2015;9:1251-60.

[25] Mazzaro C, Zorat F, Comar C, et al. Interferon-alpha plus ribavirin in patients with hepatitis $C$ virus positive mixed cryoglobulinemia resistant to interferon. Journal of Rheumatology 2003;30:1775-81.

[26] Cakir N, Nuri Pamuk O, Umit H, et al. Successful treatment with Adefovir of one patient whose cryoglobulinemic vasculitis relapsed under Lamivudine therapy and who was diagnosed to have HBV virologic breakthrough with YMDD mutations. Internal Medicine 2006;45:1213-5.

[27] Tarantino A, Campise M, Banfi C, et al. Long term predictors of survival in 209 essential mixed cryoglobulinemic glomerulonephritis. Kidney International $1995 ; 47: 618-23$

[28] Mazzaro C, Panarello G, Tesio F, et al. Hepatitis C virus risk: a hepatitis C virus related syndrome. Journal of Internal Medicine 2000;247:535-45.

[29] Elewa U, Sandri A, Kim R, et al. Treatment of hepatitis B virus-associated nephropaty. Nephron Clinical Practice 2011;119:c41-9.

[30] Yang Y, Xiong Q Zhao W, et al. Complete remission of hepatitis B virus related membranous nephropaty after Entecavir monotherapy. Clinics and Research in Hepatology and Gastroenterology 2012;36:e89-92.

[31] Quartuccio L, Soardo G, Romano G, et al. Rituximab treatment for glomerulonephritis in HCV-associated mixed cryoglobulinaemia: efficacy and safety in the absence of steroids. Rheumatology 2006;45:842-6.

[32] Roccatello D, Baldovino S, Rossi D, et al. Long-term effects of anti-CD20244 monoclonal antibody treatment of cryoglobulinaemic glomerulonephritis. Nephrology, Dialysis, Transplantation 2004;19:3054-61. 
[33] European Association for the Study of the Liver. EASL clinical practice guidelines: management of chronic hepatitis B virus infection. Journal of Hepatology 2012;57:167-85. Epub 2012 March 20: No abstract available. Erratum in: Journal of Hepatology 2013;58:201. Janssen, Harry [corrected to Janssen, Harry LA].

[34] Tassopoulos NC, Volpes R, Pastore G, et al. Efficacy of lamivudine in patients with hepatitis B e antigen-negative/hepatitis B virus DNA-positive (precore mutant) chronic hepatitis B. Lamivudine Precore Mutant Study Group. Hepatology 1999;29:889-96.

[35] Hadziyannis SJ, Tassopoulos NC, Heathcote EJ, et al. Adefovir Dipivoxil 438 Study Group. Adefovir dipivoxil for the treatment of hepatitis B e antigen-negative chronic hepatitis B. New England Journal of Medicine 2003;348:800-7. Erratum in: New England Journal of Medicine 2003;348:1192

[36] Marcellin P, Bonino F, Lau GK, et al. Peginterferon alfa-2a in HBeAgnegative Chronic Hepatitis B Study Group. Sustained response of hepatitis B e antigen-negative patients 3 years after treatment with peginterferon alpha-2a. Gastroenterology 2009;136:2169-79.

[37] Marcellin P, Piratvisuth T, Brunetto M, et al. Increasing rates of HBsAg clearance and seroconversion in patients with $\mathrm{HBeAg}$-negative disease treated with peginterferon alfa- $2 \mathrm{~A} \pm$ lamivudine: results of 5-year post-treatment follow up. Journal of Hepatology 2009;50(Suppl. 1):S336.

[38] Marcellin P, Buti M, Gane EJ, et al. Five years of treatment with tenofovir DF (TDF) for chronic hepatitis B (CHB) infection is associated with sustained viral suppression and significant regression of histological fibrosis and cirrhosis. Hepatology 2011;54(Suppl. 1):1011A.

[39] Hadziyannis SJ, Tassopoulos NC, Heathcote EJ, et al. Adefovir Dipivoxil 438 Study Group. Long-term therapy with adefovir dipivoxil for HBeAg-negative chronic hepatitis B for up to 5 years. Gastroenterology 2006;131:1743-51.

[40] D'Amico E, Pace-Palitti V, Di Lembo E, et al. Successful treatment of hepatitis B virus infection and related cryoglobulinemic purpura with nucleotide analogues. Clinical and Experimental Rheumatology 2013;31:1559. 